\title{
Dual Roles of Metal-Organic Framework as Nanocarriers for miRNA Delivery and Adjuvants for Chemodynamic Therapy
}

Huaixin Zhao, ${ }^{\dagger, \#}$ Taotao Li, ${ }^{\dagger, \#}$ Chi Yao $,{ }^{\dagger} \mathrm{Zi} \mathrm{Gu},{ }^{\dagger}$ Chunxia Liu,,${ }^{\dagger}$ Jiahe Li, ${ }^{\S}$ Dayong Yang ${ }^{\dagger, *}$

${ }^{\dagger}$ Frontiers Science Center for Synthetic Biology, Key Laboratory of Systems Bioengineering (MOE), School of Chemical Engineering and Technology, Tianjin University, Tianjin, 300350, P.R. China.

${ }^{\ddagger}$ School of Chemical Engineering and Australian Centre for NanoMedicine, University of New South Wales, Sydney, NSW 2052, Australia.

${ }^{\S}$ Department of Bioengineering, Northeastern University, Boston, MA, 02115, United States.

*Corresponding author. E-mail: dayong.yang@tju.edu.cn.

${ }^{\#}$ Huaixin Zhao and Taotao Li contributed equally to this work. 


\section{Table of Contents}

1. Materials and Reagents

2. Instruments and Characterization

3. Experimental Section

4. Figures and Figures Captions

Figure S1-S34 


\section{Materials and Reagents}

Zinc nitrate hexahydrate (AR) and 2-methylimidazole (2-MeIM, AR) were purchased from Aladdin Chemistry Co., Ltd. (Shanghai, China). N, N-dimethylformamide (DMF, AR) and ethanol were obtained from Fuyu Fine Chemical Co., Ltd. (Tianjin, China). All the solutions were prepared with DEPC-treated water. RPMI 1640 medium, fetal bovine serum (FBS), polyvinylidene fluoride (PVDF) membrane with pore size of $0.22 \mu \mathrm{m}$, BCA Protein Assay Kit, total RNA Extraction Kit, 3-(4,5-dimethylthiazol-2-yl)-2,5-diphenyltetrazolium bromide (MTT), 4',6-diamidino-2-phenylindole (DAPI) were provided by Solarbio Science \& Technology Co., Ltd. (Beijing, China). The antibodies against Bcl-2, $\beta$-actin, horseradish peroxidase (HRP)-labeled goat anti-mouse IgG were purchased from Cell Signaling Technology (Shanghai, China). Fasting RT Kit with gDNase was bought from Tiangen Biotech Co., Ltd. (Beijing, China). miR-34a-m, 5-FAM-labeled miR-34a-m and 5-Cy5-labeled miR34a-m were bought from Sangon Biotech Co., Ltd. (Shanghai, China). These HPLC purified RNA sequences are listed below:

miR-34a: sense: 5'-UGGCAGUGUCUUAGCUGGUUGU-3'; antisense: 5'AACCAGCUAAGACACUGCCAUU-3'.

FAM-miR-34a-m sense: 5'-FAM-UGGCAGUGUCUUAGCUGGUUGU-3'; antisense: 5'AACCAGCUAAGACACUGCCAUU-3'.

Cy5-miR-34a: sense: 5'-Cy5-UGGCAGUGUCUUAGCUGGUUGU-3'; antisense: 5'AACCAGCUAAGACACUGCCAUU-3'.

Negative control miRNA (NC): sense: 5'-UUCUCCGAACGUGUCACGUTT-3'; antisense: 5'-ACGUGACACGUUCGGAGAATT-3'.

These relevant DSL purified primer sequences for Bcl-2 (target protein) and $\beta$-actin (internal reference protein) are listed below:

Bcl-2 forward primer: 5'-GACTTCGCCGAGATGTCCAG-3'.

Bcl-2 reverse primer: 5'-GAACTCAAAGAAGGCCACAATC-3'. 
$\beta$-actin forward primer: 5'-ATCGTGCGTGACATTAAGGAGAAG-3' .

$\beta$-actin reverse primer: 5'-AGGAAGGAAGGCTGGAAGAGTG-3'.

\section{Instruments and Characterization}

Scanning electron microscopy (SEM) image (Hitachi-S4800 FESEM, Japan), transmission electron microscopy (TEM) image and the energy dispersive spectroscopy (EDS) mapping picture (JEM-2100F Flash, Japan) at an acceleration voltage of $200 \mathrm{kV}$ were used to investigate the morphology. The hydrodynamic size and zeta potential were performed on a Zetasizer Nano ZS90 unit (Malvern, the United Kingdom). Fourier transform infrared (FT-IR) spectra were measured by a Bruker Tensor 27 spectrometer (Nikon, Germany). Fluorescent images were obtained using a Biotech Ti-E inverted fluorescence microscope (Nikon, Japan). In vivo luminescence image was acquired on a Berthold Night OWL LB 983 NC100 Imaging system (Berthold, Germany). The absorbance and fluorescence intensity were acquired by a Biotech SYNERGY H1 microplate reader. The X-ray diffractometer D8-Focus with $\mathrm{Cu} \mathrm{K} \alpha$ radiation (Bruker, Germany) was used to collect the powder XRD data.

\section{Experimental Section}

Adsorption of Cy5-miR-34a-m on ZIF-8. Briefly, Cy5-miR-34a-m was first dissolved in DEPC-treated water. Then, $0.275 \mathrm{mg}$ of ZIF-8 was dispersed in $205 \mu \mathrm{L}$ of $21 \mu \mathrm{g}$ Cy5-miR-34a$\mathrm{m}$ solution. And the mixture was shaked on the shaker at $500 \mathrm{rpm}, 30 \mathrm{~min}$. After that, the mixture was collected by centrifuging at $12000 \mathrm{rpm}$ for $10 \mathrm{~min}$ to obtain the precipitates of Cy5-miR-34a-m@ZIF-8, and the fluorescence intensity of free Cy5-miR-34a-m was measured by a microplate reader. The loading capacity of Cy5-miR-34a-m on ZIF-8 was $36 \mu \mathrm{g}$ Cy5-miR34a/mg ZIF-8. The loading capacity was calculated as follows:

$$
\begin{gathered}
\text { Loading efficiency }=\frac{\mathrm{I}_{\mathrm{Cy} 5-\mathrm{miR}-34 \mathrm{a}-\mathrm{m}, \text { initial }}-\mathrm{I}_{\mathrm{Cy} 5-\mathrm{miR}-34 \mathrm{a}-\mathrm{m}, \text { residual }}}{\mathrm{I}_{\mathrm{Cy} 5-\mathrm{miR}-34 \mathrm{a}-\mathrm{m}, \text { initial }}} \times 100 \% \\
\text { Loading capacity }=\frac{0.021 \mathrm{mg} \times \text { Loading efficiency }}{0.275 \mathrm{mg}}
\end{gathered}
$$


where Cy5-miR-34a $\mathrm{a}_{\text {initial }}$ and Cy5-miR-34aresidual were the fluorescence intensity of Cy5-miR34a-m before and after load.

In vitro $\mathrm{pH}-$-responsive miR-34a-m release experiments. To investigate $\mathrm{pH}$-responsive miR34a-m release procedure, $50 \mu \mathrm{L}$ of PBS (10 mM, pH 5.5) and $50 \mu \mathrm{L}$ of PBS (10 mM, pH 7.4) were respectively added into $190 \mu \mathrm{L}$ of $207 \mu \mathrm{g}$ Cy5-miR-34a-m@ZIF-8. After incubation at 37 ${ }^{\circ} \mathrm{C}$ for a period of time $(0-7 \mathrm{~h})$, the fluorescence intensity of the supernatant was measured by a microplate reader. The cumulative released Cy5-miR-34a-m from the Cy5-miR-34a-m@ZIF-8 at $\mathrm{pH} 5.5$ and 7.4 were calculated.

miR-34a-m stability of miR-34a-m@ZIF-8. miR-34a-m stability of miR-34a-m@ZIF-8 was evaluated by polyacrylamide gel electrophoresis (PAGE). $3 \mu \mathrm{L}$ of FBS was added into $9 \mu \mathrm{L}$ of $35.3 \mu \mathrm{g}$ miR-34a-m@ZIF-8. The mixture was incubated at $37{ }^{\circ} \mathrm{C}$ for $1,3,5 \mathrm{~h}$, and then $8 \mu \mathrm{L}$ of PBS (10 mM, pH 5.5) was added to split ZIF-8. miR-34a-m solution including the same concentration as the miR-34a-m@ZIF-8 was as control. Next, the miR-34a-m stability was evaluated on $20 \%$ PAGE at $120 \mathrm{~V}, 90 \mathrm{~min}$.

ROS determination with MB. $25 \mathrm{mM} \mathrm{NaHCO} / 5 \% \mathrm{CO}_{2}$ buffer solution containing 100 $\mu \mathrm{g} / \mathrm{mL} \mathrm{MB}, 25 \mathrm{mM} \mathrm{H}_{2} \mathrm{O}_{2}$, and different concentrations of ZIF-8 and $\mathrm{NH}_{2}-\mathrm{MIL}-53$ (Fe) were added. Then, the mixture was incubated at $37^{\circ} \mathrm{C}$ for $4 \mathrm{~h}$, and the absorbance change of $\mathrm{MB}$ at $665 \mathrm{~nm}$ was measured. The degradated percentage of MB was calculated as follows: (A $\mathrm{A}_{\text {control - }}$ A)/A $\mathrm{A}_{\text {control }} \mathrm{X} 100 \%$

Cell culture. Human breast cancer cells (MDA-MB-231) were provided by the School of Medicine of Nankai University. The cells were cultured with RPMI 1640 medium, which was supplemented with $10 \%$ fetal bovine serum (FBS), $100 \mu \mathrm{g} / \mathrm{mL}$ streptomycin, $100 \mathrm{U} / \mathrm{mL}$ penicillin and $50 \mu \mathrm{g} / \mathrm{mL}$ gentamicin. The cells were kept in a $100 \%$ humidified atmosphere containing $5 \% \mathrm{CO}_{2}$ at $37{ }^{\circ} \mathrm{C}$, following the protocol of American Type Culture Collection (ATCC). 
Intracellular uptake of FAM-miR-34a-m@ZIF-8. MDA-MB-231 cells $\left(7 \times 10^{4}\right.$ cells $)$ were seeded into glass-bottomed microscope dishes and incubated for $12 \mathrm{~h}$ to allow the cells to attach onto the glass bottom. Then, $20 \mu \mathrm{L}$ of $25.9 \mu \mathrm{g}$ miR-34a-m@ZIF-8 were added to microscope dishes. After incubation for $4 \mathrm{~h}$, the cells were washed three times with PBS and fixed with $4 \%$ fixative solution (paraformaldehyde) for $20 \mathrm{~min}$. Following this, the cell nuclei were stained with DAPI $(1 \mu \mathrm{g} / \mathrm{mL})$. After $15 \mathrm{~min}$, the cells were washed twice with PBS and the photos were recorded by CLSM.

Subcellular localization of FAM-miR-34a-m@ZIF-8. To further monitor the process of cell internalization, the subcellular localization was observed by CLSM. $20 \mu \mathrm{L}$ of $25.9 \mu \mathrm{g}$ miR-34am@ZIF-8 were added to the glass-bottomed microscope dishes seeded MDA-MB-231 cells $\left(7 \times 10^{4}\right.$ cells) for $1 \mathrm{~h}$. Then the cells were stained with Lyso Tracker Red for $1 \mathrm{~h}$. Next protocols were the same as intracellular uptake.

Intracellular ROS generation. MDA-MB-231 cells $\left(7 \times 10^{4}\right.$ cells $)$ were seeded in six-well plates overnight. Then, $20 \mu \mathrm{L}$ of $25.9 \mu \mathrm{g}$ miR-34a-m@ZIF-8 were added to MDA-MB-231 cells. After $4 \mathrm{~h}$, the treated cells were incubated with $10 \mu \mathrm{M}$ of 2,7-dichlorofluorescein diacetate (DCFH-DA), which was the fluorescent probe for measuring intracellular ROS generation. Fluorescent imaging of DCF was monitored using fluorescence microscopy with $488 \mathrm{~nm}$ excitation and $525 \mathrm{~nm}$ emission.

Cell viability test. First, the MTT assay was performed to assess the cell viability with different treatments. MDA-MB-231 cells were planted on 96-well plates at a density of $5 \times 10^{3}$ cells per well and incubated overnight. Then the cells were respectively treated a series of ZIF-8, miR34a-m or miR-34a-m@ZIF-8 with various concentrations for $48 \mathrm{~h}$. After washing twice with PBS, $100 \mu \mathrm{L}$ of MTT $(0.5 \mathrm{mg} / \mathrm{mL})$ was added into per well and further incubated at $37{ }^{\circ} \mathrm{C}$ for $4 \mathrm{~h}$. Finally, $110 \mu \mathrm{L}$ of DMSO was added after removing the old medium. After gently shaking for $10 \mathrm{~min}$, the absorbance at $490 \mathrm{~nm}$ (OD 490) was measured by a microplate reader. The cell viability was calculated as follows: 


$$
\text { Cell viability }=\frac{O D_{\text {Treated }}-O D_{\text {Blank }}}{O D_{\text {Control }}-O D_{\text {Blank }}} \times 100 \%
$$

where the $\mathrm{OD}_{\text {Treated }}$ represents the absorbance of cells incubated with samples, $\mathrm{OD}_{\text {Control }}$ represents the absorbance of cells incubated with PBS and $\mathrm{OD}_{\text {Blank }}$ represents the absorbance of culture media with samples or PBS.

RT-qPCR analysis. MDA-MB-231 cells were planted in six-well plates at a density of $5 \times 10^{5}$ cells per well and incubated overnight. $32 \mu \mathrm{g} / \mathrm{mL}$ of ZIF-8 and $100 \mathrm{nM}$ of miR-34a-m were selected. Then the cells were respectively treated with PBS, ZIF-8, miR-34a-m and miR-34am@ZIF-8 for $48 \mathrm{~h}$. Then the cells were washed by PBS and the intracellular total RNA was extracted by total RNA Extraction Kit. mRNA was reverse transcribed by FastKing RT Kit (With gDNase). Additionally, quantitative PCR analysis was performed using the SuperReal PreMix Plus (SYBR Green). Amplification was monitored on LightCycler® 480. Data were analyzed according to the $2^{-\Delta \Delta \mathrm{Ct}}$ method.

Western blot assay. MDA-MB-231 cells were planted in six-well plates at a density of $5 \times 10^{5}$ cells per well and incubated overnight. $32 \mu \mathrm{g} / \mathrm{mL}$ of ZIF-8 and $100 \mathrm{nM}$ of miR-34a-m were selected. Then the cells were respectively treated with PBS, ZIF-8, miR-34a-m and miR-34am@ZIF-8 for $48 \mathrm{~h}$. Then the proteins were harvested by using $1 \times$ SDS Lysis Buffer and quantified by using BCA Protein Assay Kit. After dilution into the same concentration, these proteins were separated by SDS-PAGE gradient gel and transferred to the PVDF membrane. Then PVDF membrane was blocked in 5\% non-fat powdered milk and blocked with antibodies against Bcl-2 (1:1000) and $\beta$-actin (1:5000). The membrane was incubated with secondary antibody and then analyzed using an automatic chemiluminescence image system (Tanon 4600SF).

EdU assays. The 5'-Ethynyl-2'-deoxyuridine EdU assay was done using the Meilun EdU Cell Proliferation Kit with Alexa Fluor 488 (Dalian Meilun Co.,Dalian, China) according to protocols. For the proliferation assay, cells were plated in 96-well plates at $\sim 50 \%$ confluence 2 
days before the assay. Cells were incubated with a nucleoside analog of thymidine, 5-ethynyl2'-deoxyuridine (EdU), for 60 minutes, followed by fixation and staining for EdU-incorporated cells with the use of the ClickiT EdU Assay kit (Invitrogen, Carlsbad, CA). After $24 \mathrm{~h}$, cell proliferation was evaluated by EdU proliferation assay. The percentage of EdU-positive cells was quantified. Results were presented as mean $\pm \operatorname{SD}(n=6)$.

Apoptosis assays. Cell apoptosis was detected using Annexin V-FITC/PI Apoptosis Detection Kit (Solarbio, China) according to protocols. MDA-MB-231 cells were cultured at $5 \times 10^{5}$ cells per well in a six well plate overnight. Then the cells were respectively treated with PBS, ZIF-8 and miR-34a-m@ZIF-8 for 48 h. After incubation, the cells were washed with PBS and binding buffer. $8 \mu \mathrm{L}$ of Annexin V-FITC was added and incubated for $10 \mathrm{~min}$ in dark, then $8 \mu \mathrm{L}$ PI were added and incubated for $5 \mathrm{~min}$ in dark. The stained cells were then examined under fluorescent microscope.

Scratch test. MDA-MB-231 cells were planted in six-well plates at a density of $7 \times 10^{5}$ cells per well and incubated overnight, and a scratch was made in a straight line. $20 \mu \mathrm{L}$ of $25.9 \mu \mathrm{g}$ miR34a-m@ZIF-8 were added into cells as the experimental group while PBS treated as control group, then the images were recorded at $0 \mathrm{~h}$ and $24 \mathrm{~h}$. The migration rate was calculated according to the following formula:

$$
\mathrm{R}_{\mathrm{M}}=\frac{\mathrm{W}_{\mathrm{i}}-\mathrm{W}_{\mathrm{f}}}{\mathrm{t}}
$$

where the $R_{M}$ represents the rate of cell migration $(\mu \mathrm{m} / \mathrm{h}), \mathrm{W}_{\mathrm{i}}$ represents the initial wound width $(\mu \mathrm{m}), \mathrm{W}_{\mathrm{f}}$ represents the final wound width $(\mu \mathrm{m})$ and $\mathrm{t}$ represents the duration of migration $(\mathrm{h})$. Wound width was calculated as the average distance between the edges of the scratch $(n=3)$. Control: $6.5 \mu \mathrm{m} / \mathrm{h}$; miR-34a-m@ZIF-8: $2.0 \mu \mathrm{m} / \mathrm{h}$.

Hemolysis test. The fresh blood of mice was centrifuged at $5000 \mathrm{rpm}$ for $3 \mathrm{~min}$ at $4{ }^{\circ} \mathrm{C}$, and the supernatant was discarded. The collected red blood cells (RBCs) were washed for three times with normal saline $(0.9 \% \mathrm{NaCl}$ solution) and resuspended in normal saline. $200 \mu \mathrm{L}$ of $\mathrm{RBCs}$ 
suspension was mixed with $800 \mu \mathrm{L}$ of miR-34a-m@ZIF-8 $(200,400,600 \mu \mathrm{g} / \mathrm{mL}), 800 \mu \mathrm{L}$ of normal saline (negative control) and $800 \mu \mathrm{L}$ of water (positive control), respectively. Then the mixtures were incubated at $37^{\circ} \mathrm{C}$ for $30 \mathrm{~min}$, followed by centrifuging to collect the supernatants at $5000 \mathrm{rpm}$ for $3 \mathrm{~min}$. The absorbance value of the supernatants at $570 \mathrm{~nm}$ were measured. The hemolysis percent of RBCs was analyzed according to the following equation:

$$
\text { Hemolysis }=\frac{A_{\text {Sample }}-A_{\text {Negative control }}}{A_{\text {Positive control }}-A_{\text {Negative control }}} \times 100 \%
$$

where the Asample represents the absorbance of RBCs incubated with miR-34a-m@ZIF-8, APositive control represents the absorbance of RBCs incubated with water and $\mathrm{A}_{\text {Negative control }}$ represents the absorbance of RBCs incubated with normal saline.

Animals and tumor model. Animal experiments were approved by ethics committee of Tianjin University in compliance with the law on experimental animals. Kuning mice (5-6 week old) and Balb/c female nude mice (6-7 week old) were bought from Beijing Huafukang Bioscience Co. Ltd. (Beijing, China). The MDA-MB-231 tumor-bearing mice were subcutaneously inoculated with $1 \times 10^{6}$ cells on the right back of the hind leg region and randomly divided into six groups. The control group were injected with $100 \mu \mathrm{L}$ PBS $(10 \mathrm{mM}, \mathrm{pH}$ 7.4). The treated group were respectively injected with $100 \mu \mathrm{L}$ of ZIF-8 (15 mg per kg mice) and miR-34a-m (0.5 mg per kg mice) by intravenously injection. Tumor volume was calculated according to the following formula:

$$
\mathrm{V}=\frac{\left(\mathrm{L} \times \mathrm{W}^{2}\right) \times \pi}{6}
$$

( $\mathrm{L}$ and $\mathrm{W}$ are the longest and shortest diameters of the tumor, respectively).

The tumor growth inhibition ratios of the tumors were calculated as follows ${ }^{[2]}$ :

$$
\text { Inhibition }=\frac{V a-V b}{\mathrm{Va}} \times 100 \%
$$

$V_{a}$ referred to the relative tumor volume of control group while $V_{b}$ referred to the relative tumor volume of experimental group. 
Toxity evaluatin. Six Kunming mice were equally divided into two groups. The experimantal groups were treated with miR-34a-m@ZIF-8 (15.5 mg per kg mice) via intravenously injection and sacrificed at 14 days. The control group was treated with PBS via intravenously injection. The body weights of mice were monitored to explore the in vivo physiological influences of the miR-34a-m@ZIF-8 towards organism. Blood was harvested from the two groups of mice. The blood biochemical parameters were carried out. The major organs (heat, liver, spleen, lung and kidney) were extracted and were kept in formaldehyde (10\%) and stained with hematoxylin and eosin $(\mathrm{H} \& \mathrm{E})$ for histopathologic analysis.

Live animal imaging and biodistribution. MDA-MB-231 tumor-bearing mice were intravenously injected with Cy5-miR-34a-m@ZIF-8 (15.5 mg per $\mathrm{kg}$ mice). The in vivo fluorescent images were obtained by the imaging system at 1, 3, 6 and $24 \mathrm{~h}$ post-administration to evaluate the biodistribution of Cy5-miR-34a-m@ZIF-8 in different organs. At 24 h postinjection, the mice were euthanized to obtain the internal organs (heart, liver, spleen, lung, kidney) and tumor for ex vivo imaging.

In vivo tumor growth inhibition. The MDA-MB-231 tumor-bearing mice were randomly divided into six groups $(n=5)$ and intravenously injected with the following samples: (a) PBS, (b) miR-34a-m, (c) ZIF-8, (d)NC@ZIF-8, (e)miR-34a+ZIF-8. (f) miR-34a-m@ZIF-8. The ZIF8 concentration was $15 \mathrm{mg}$ per $\mathrm{kg}$ mice and miR-34a-m concentration was $0.5 \mathrm{mg}$ per $\mathrm{kg}$ mice. Subsequently, the tumor volume and body weight were recorded every three days. At the 21 st day, mice were sacrificed and tumors were picked to record the final weight.

Statistical analysis. Statistical was analyzed by a three-sample Student's $t$ test at a significance level of $\mathrm{p}<0.5(*), \mathrm{p}<0.01(* *)$ and $\mathrm{p}<0.001(* * *)$. 


\section{Figures and Figures Captions}
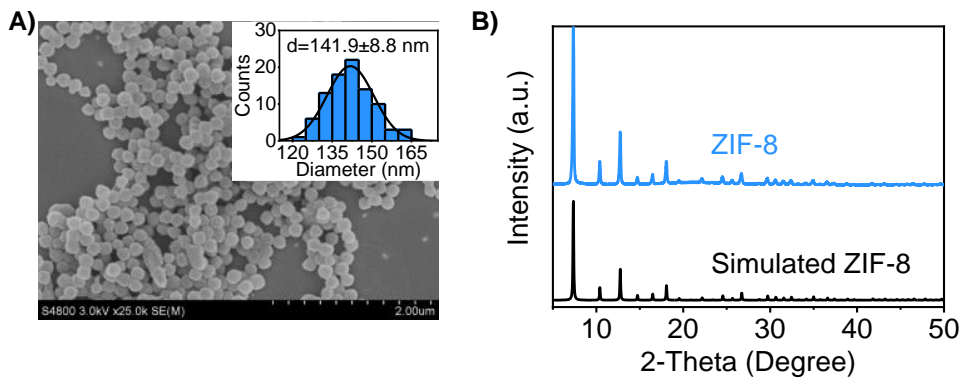

Figure S1. A) SEM image of ZIF-8. Insert: Corresponding diameter statistics. B) XRD patterns of ZIF-8 and simulated ZIF-8.

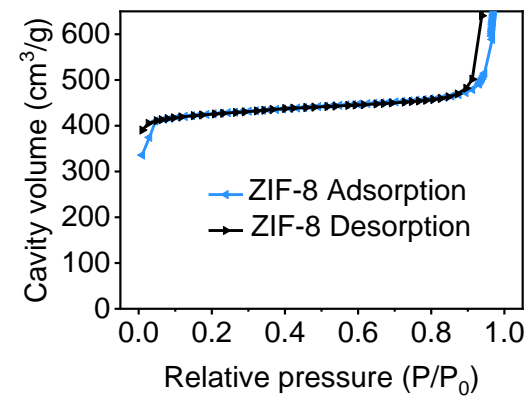

Figure S2. Nitrogen adsorption isotherms of ZIF-8 after treatment at $100{ }^{\circ} \mathrm{C}$ in air.

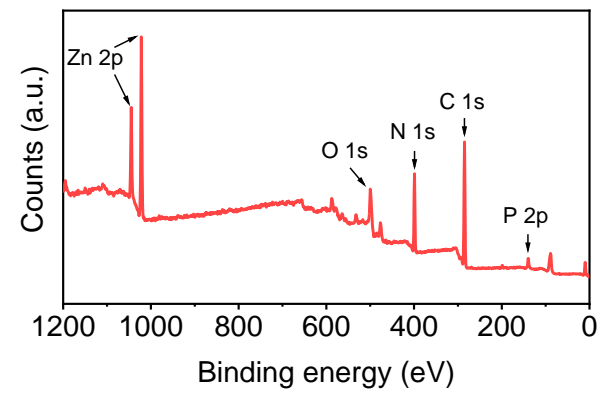

Figure S3. XPS survey spectra of the miR-34a-m@ZIF-8. 

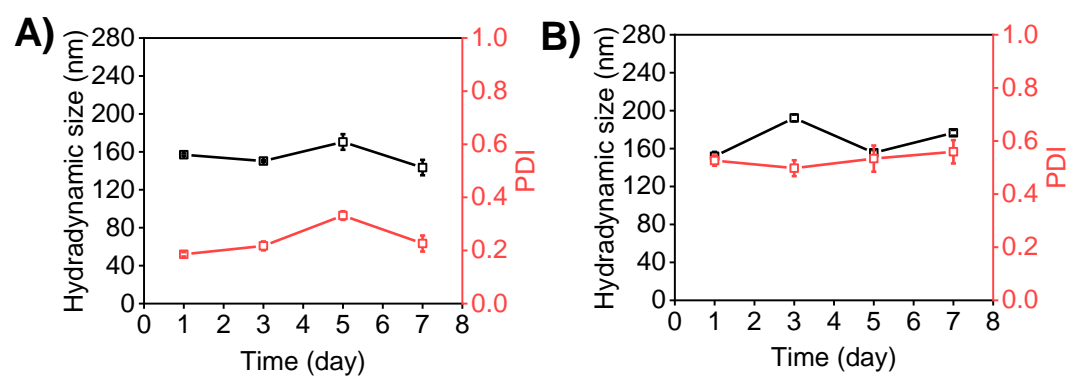

Figure S4. Long-term stability of miR-34a-m@ZIF-8 was investigated by determining the hydrodynamic diameter (black) and polydispersity index (red) of miR-34a-m@ZIF-8 (5 $\mu \mathrm{g} \cdot \mathrm{mL}^{-1}$ ), which were incubated with water (A) and culture medium $+10 \%$ FBS (B) for 7 days.

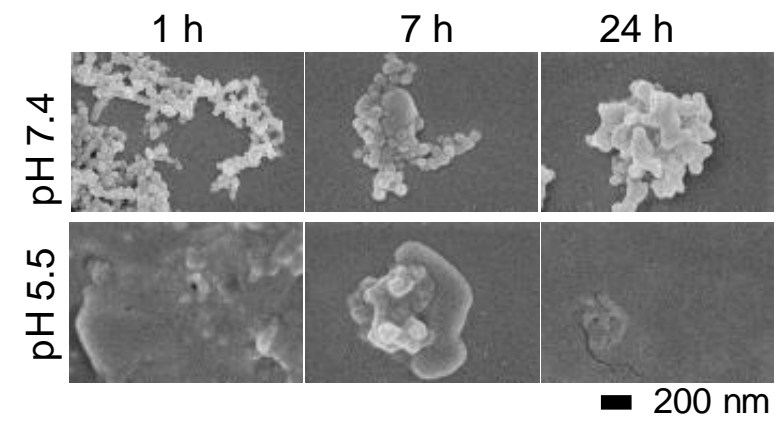

Figure S5. SEM images of miR-34a-m@ZIF-8 treated with PBS (pH 7.4 and 5.5).

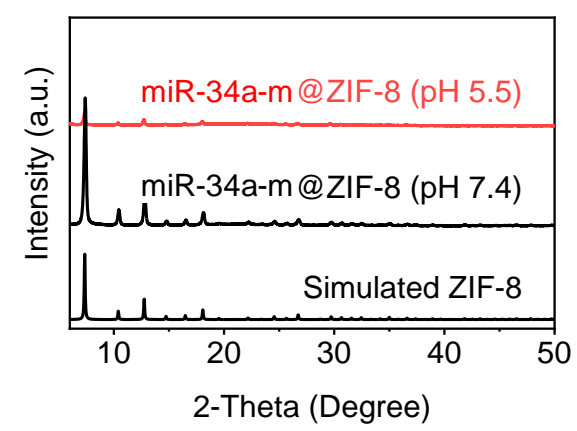

Figure S6. XRD patterns of miR-34a-m@ZIF-8 treated with PBS buffer (pH 5.5 and 7.4) for 1 hour. 


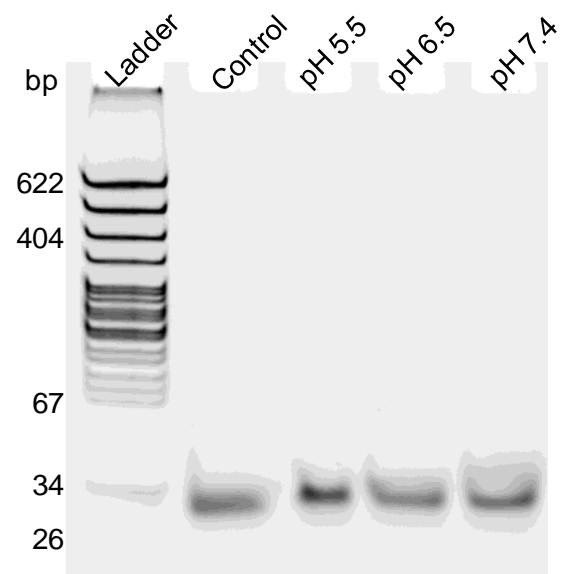

Figure S7. The stability of miR-34a-m incubated in PBS buffer ( $\mathrm{pH} 5.5,6.5$ and 7.4) verified by the polyacrylamide gel electrophoresis.

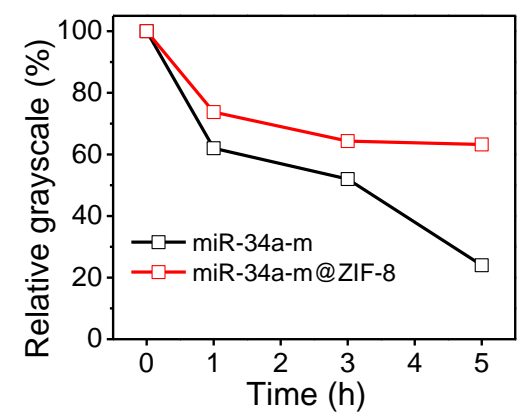

Figure S8. Quantification of band intensities of polyacrylamide gel electrophoresis image of the serum stability evaluation.

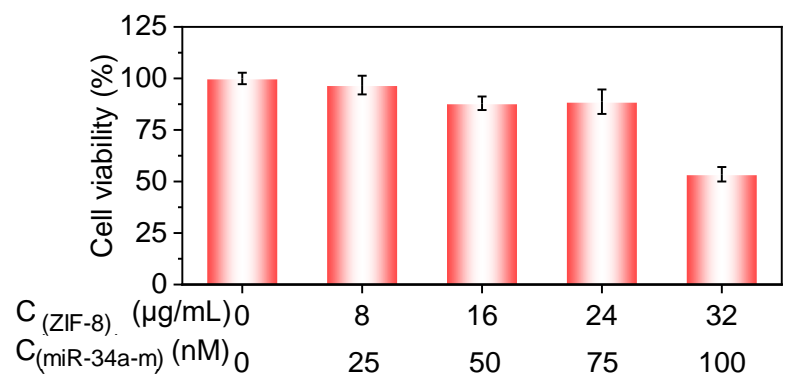

Figure S9. Cell viability of MDA-MB-231 cells incubated with different miR-34a-m@ZIF-8 concentration by MTT assay. 


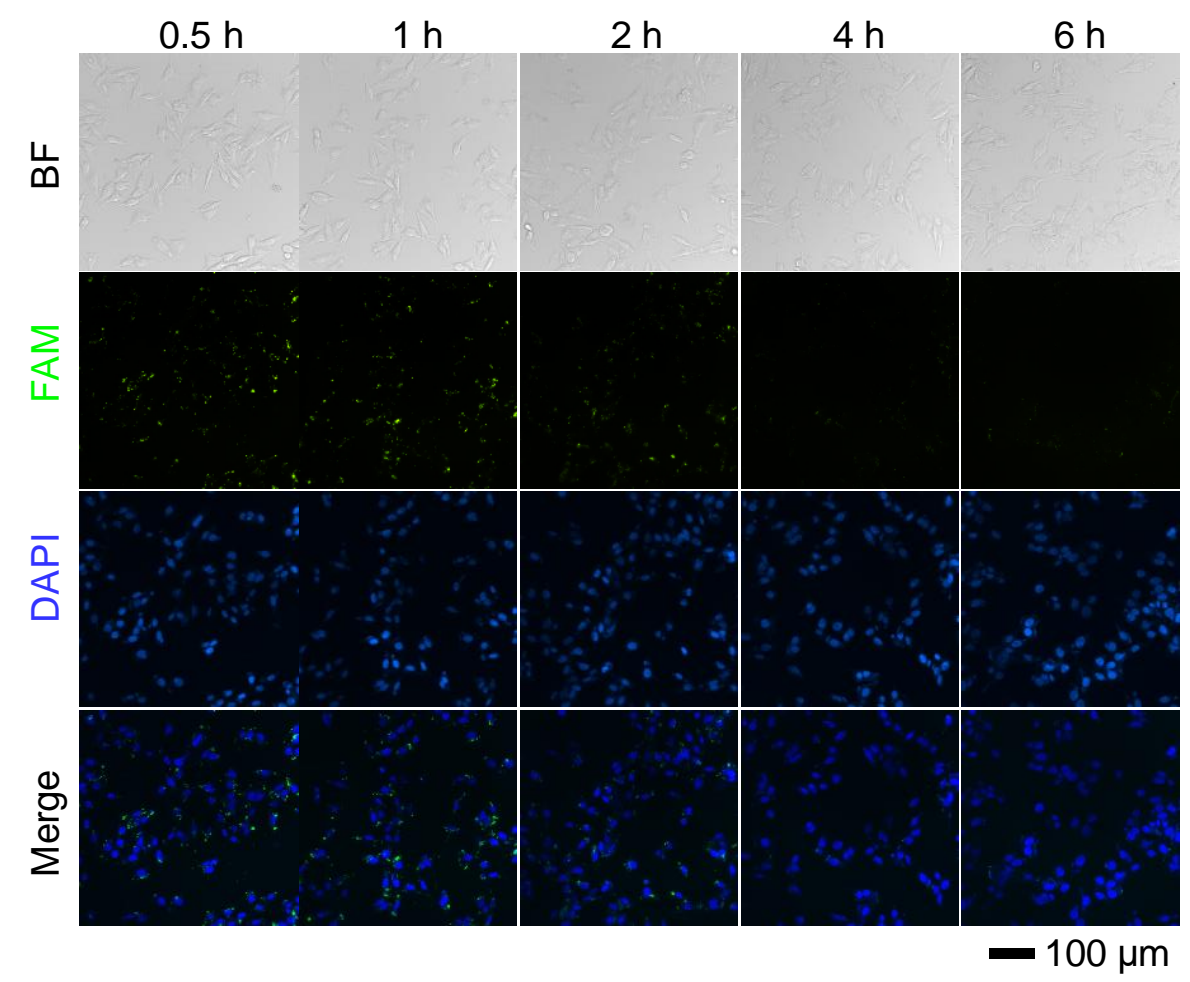

Figure S10. Microscopy images of MDA-MB-231 cells incubated with FAM-miR-34am@ZIF-8 for different time. 


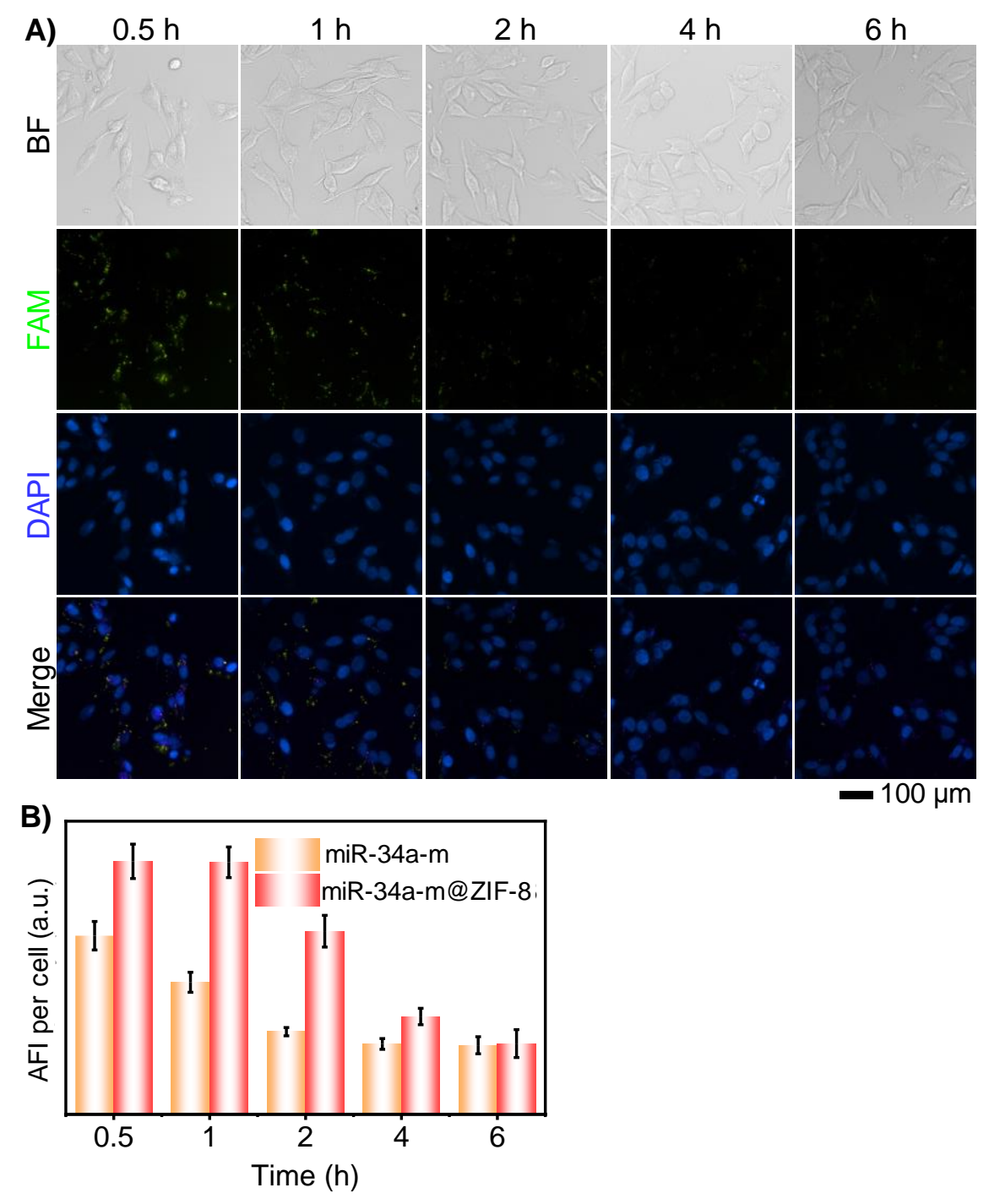

Figure S11. (A) Microscopy images of MDA-MB-231 cells incubated with FAM-miR-34a-m for different time. (B) Corresponding average fluorescence intensity (AFI) per cell of MDAMB-231 cells incubated with FAM-miR-34a-m (Figure S11A) and FAM-miR-34a-m@ZIF-8 (Figure S10) for different time.

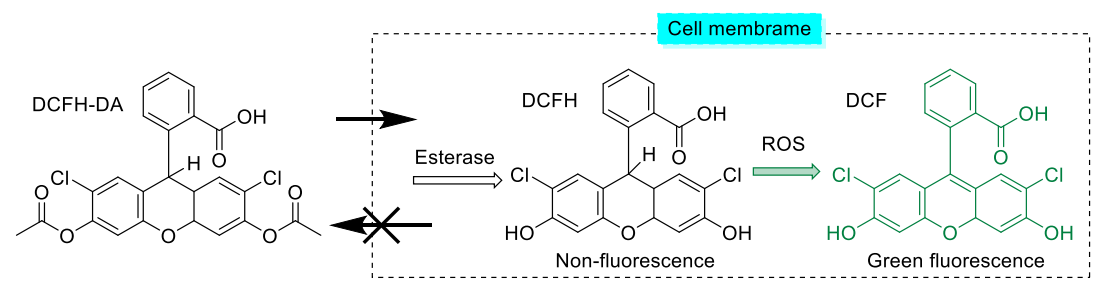

Figure S12. Schematic illustration of intracellular conversion of DCFH-DA to DCF. 


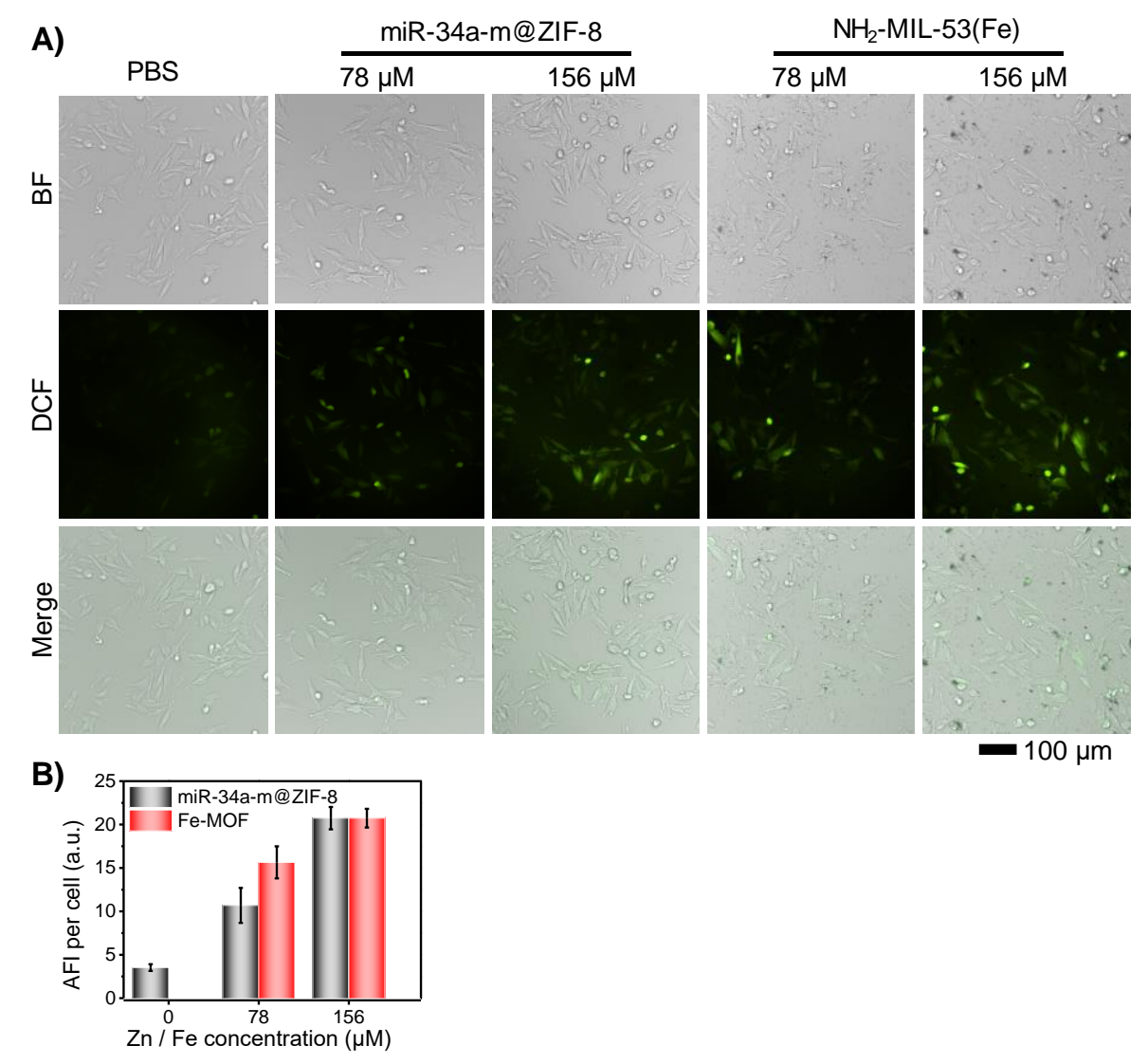

Figure S13. (A) Fluorescence microscopy images of ZIF-8 and Fe-MOF ( $\mathrm{NH}_{2}-\mathrm{MIL}-53$ (Fe)) treated MDA-MB-231 cells with DCFH-DA as ROS probe. (B) Corresponding AFI per cell of DCF fluorescence.
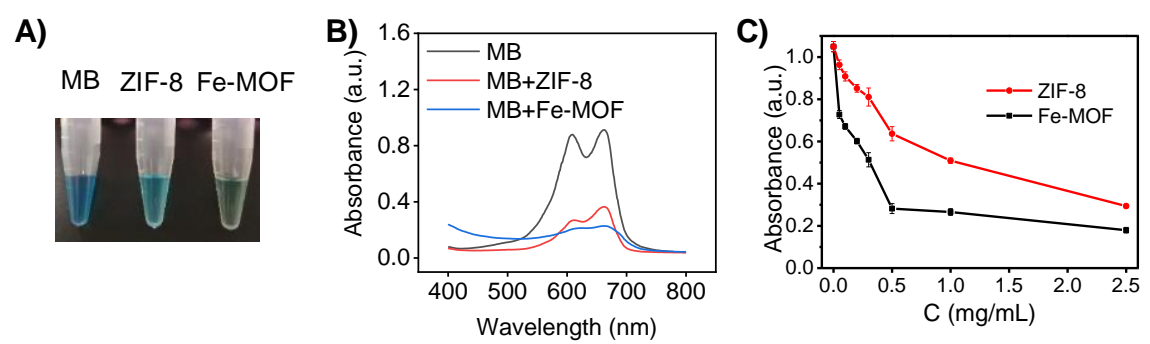

Figure S14. (A) Photo and (B) UV/Vis absorption spectra of MB after degradation by the ZIF-8 and Fe-MOF (1 mg/mL). (C) The absorbance of MB treated with different concentrations of ZIF-8 and Fe-MOF. 


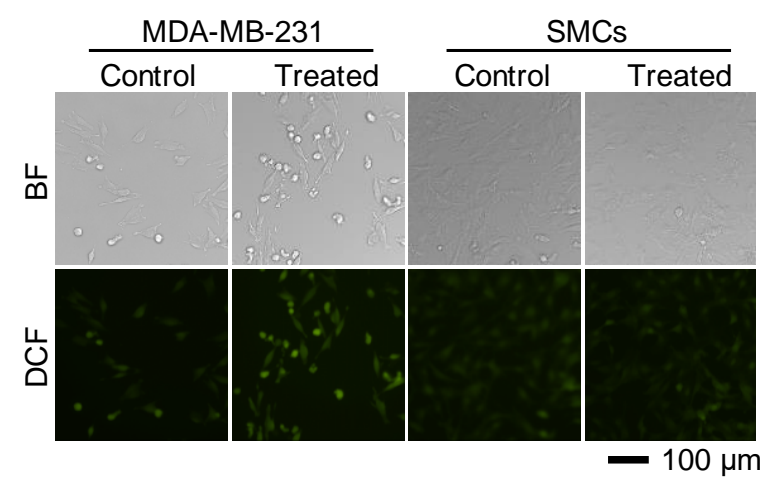

Figure S15. Microscopy images of miR-34a-m@ZIF-8 treated MDA-MB-231 and SMC cells (32 $\mu \mathrm{g} / \mathrm{mL})$ stained by DCFH-DA.

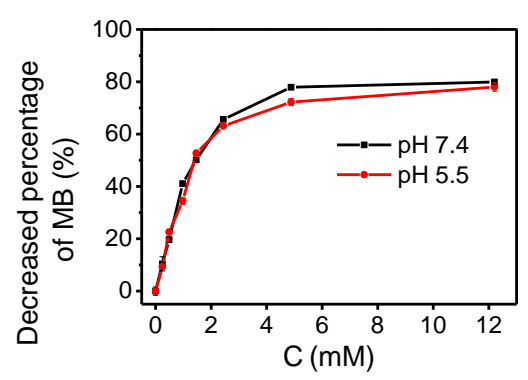

Figure S16. The decreased percentage of MB by different concentrations miR-34a-m@ZIF-8 at neutrol and acid buffer (pH 5.5 and 7.4) to simulate the normal and tumor tissue environment.
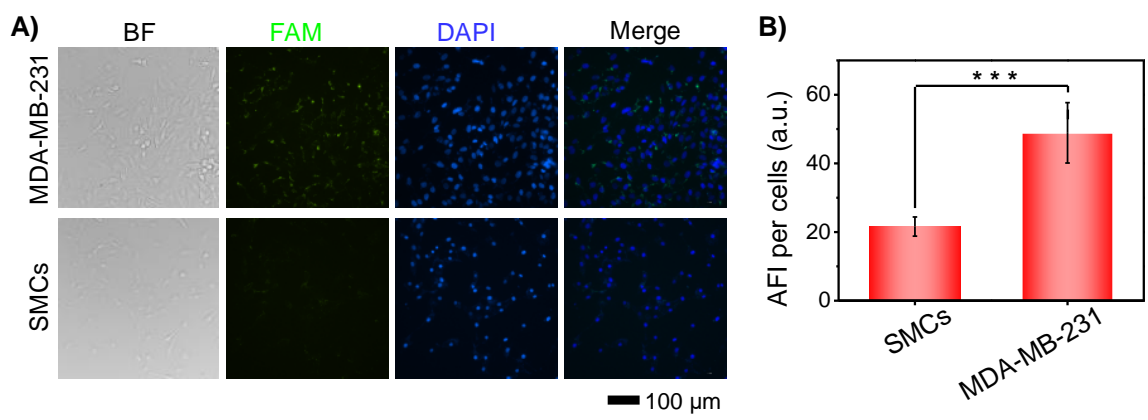

Figure S17. (A) Microscopy of cellular uptake of FAM-miR-34a-m@ZIF-8 by MDA-MB231 cancer cells and smooth muscle cells (SMCs). (B) Corresponding AFI per cell of the green channel in (A). 


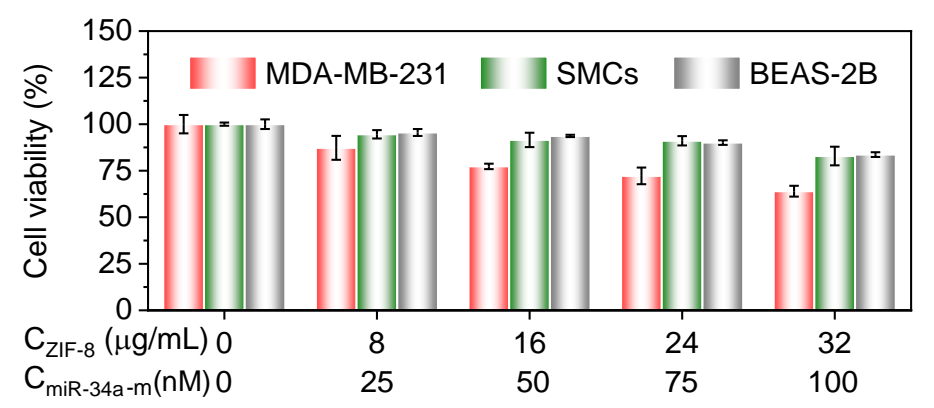

Figure S18. Cell viability of miR-34a-m@ZIF-8 on the MDA-MB-231 cancer cells and normal cells of SMCs and BEAS-2B as control groups.

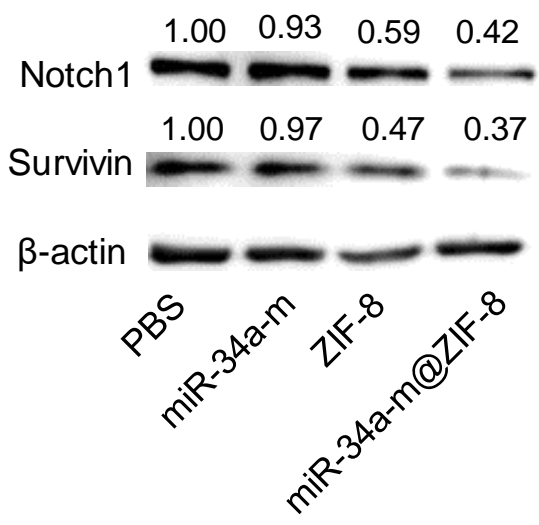

Figure S19. Western blot analysis of Notch 1 and Survivin protein in MDA-MB-231 cells treated with PBS, miR-34a-m, ZIF-8 and miR-34a-m@ZIF-8, respectively. 


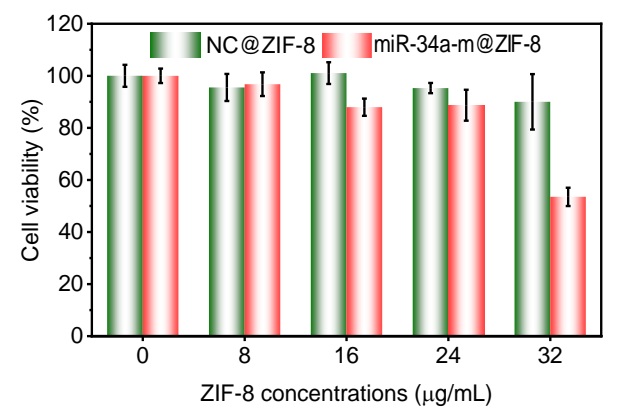

Figure S20. Cell viability of MDA-MB-231 cells incubated with different concentrations of miR-34a-m@ZIF-8 and NC@ZIF-8 tested by MTT assay. Data represent mean \pm s.d. $(n=3)$.

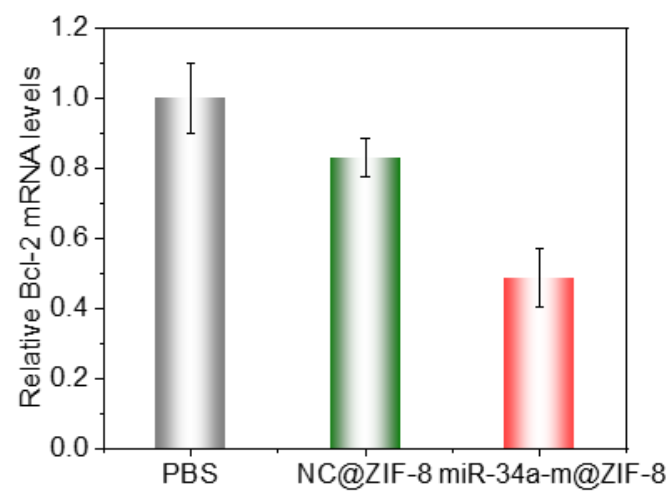

Figure S21. RT-qPCR analysis of Bcl-2 mRNA in MDA-MB-231 cells treated with PBS, NC@ZIF-8 and miR-34a-m@ZIF-8, respectively. Data represent mean \pm s.d. $(\mathrm{n}=3)$.

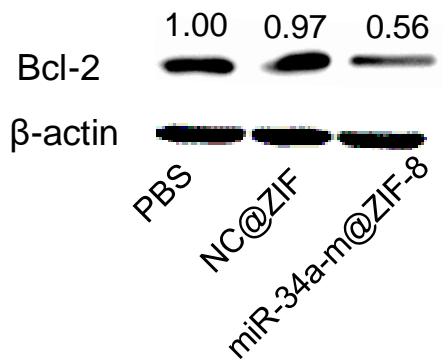

Figure S22. Western blot analysis of Bcl-2 protein in MDA-MB-231 cells treated with PBS, NC@ZIF-8 and miR-34a-m@ZIF-8, respectively. 


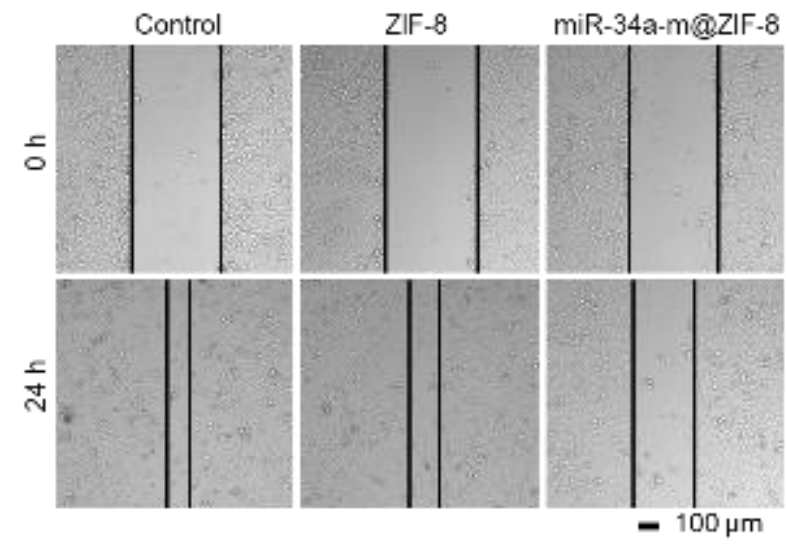

Figure S23. The microscopy images of the scratch assay of, PBS (control), ZIF-8 and miR34a-m@ZIF-8 treated MDA-MB-231 cell.

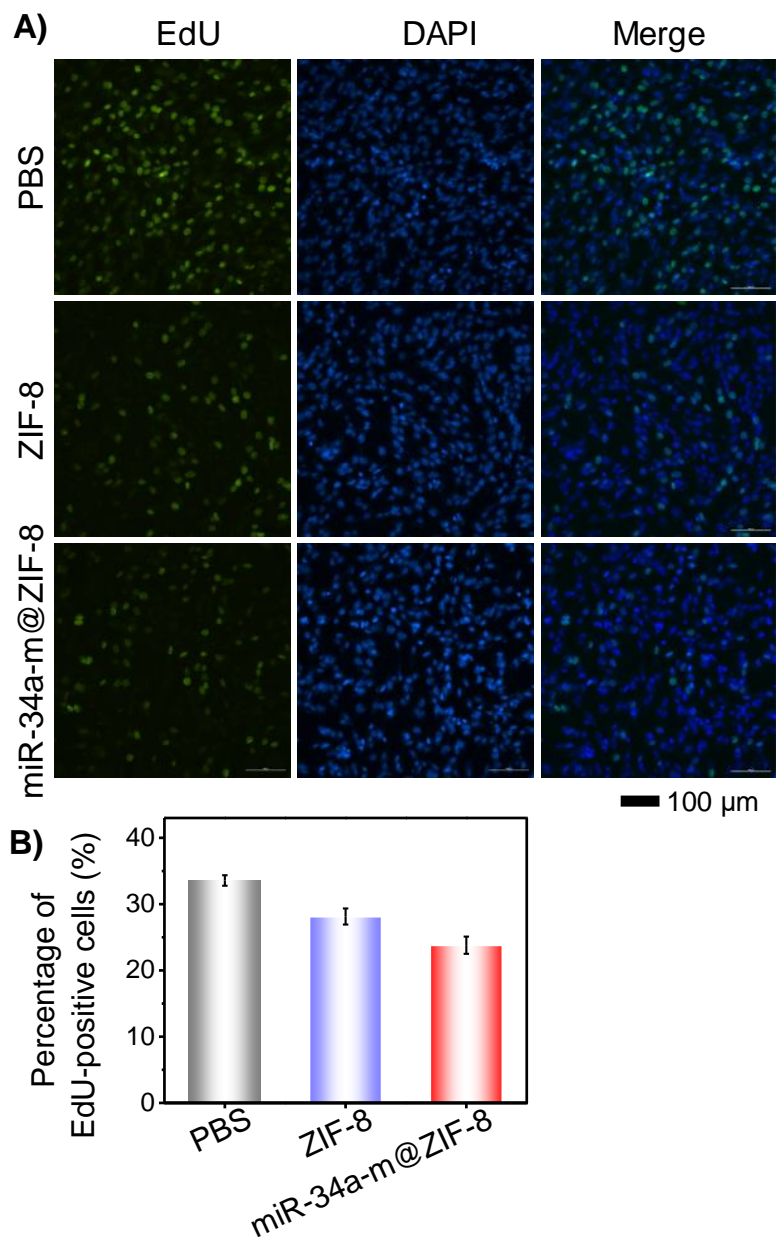

Figure S24. (A) Microscopy of EdU proliferation assay to determine cell proliferation ability.

(B) Corresponding AFI of green channel in (A). The nuclei were stained with DAPI and EdU. 


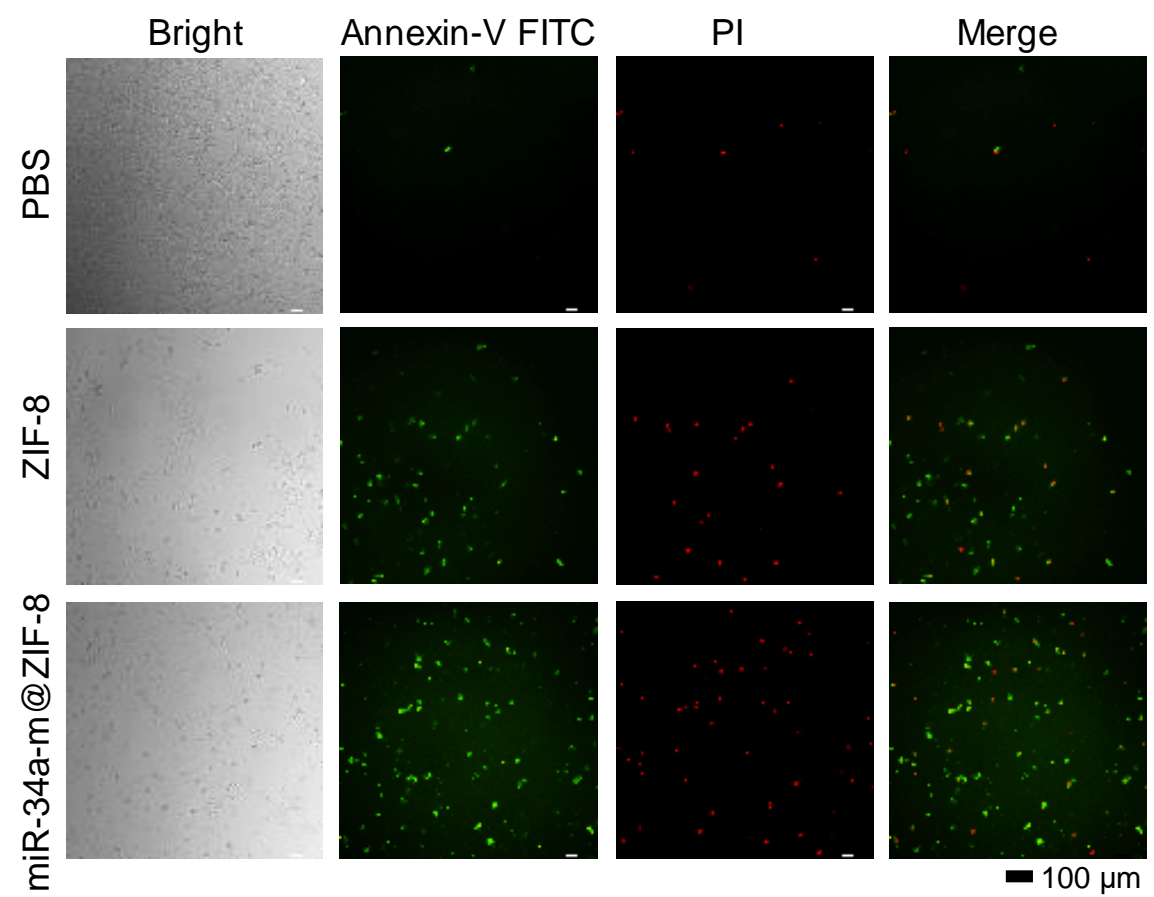

Figure S25. Fluorescent microscopic image of Annexin V-FITC (green)/PI (red) stained apoptotic MDA-MB-231 cells. Scale bar $=100 \mu \mathrm{m}$.

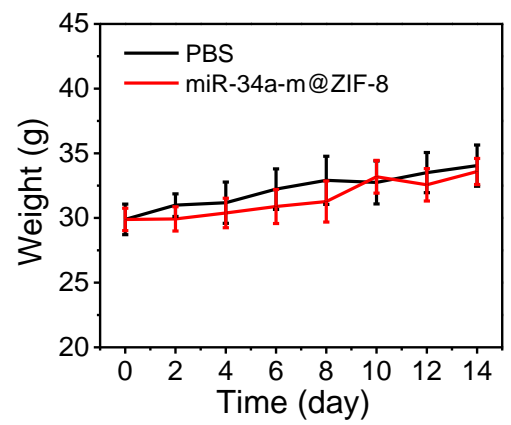

Figure S26. Bodyweight changes of miR-34a-m@ZIF-8 treated mice with PBS treated as control. 

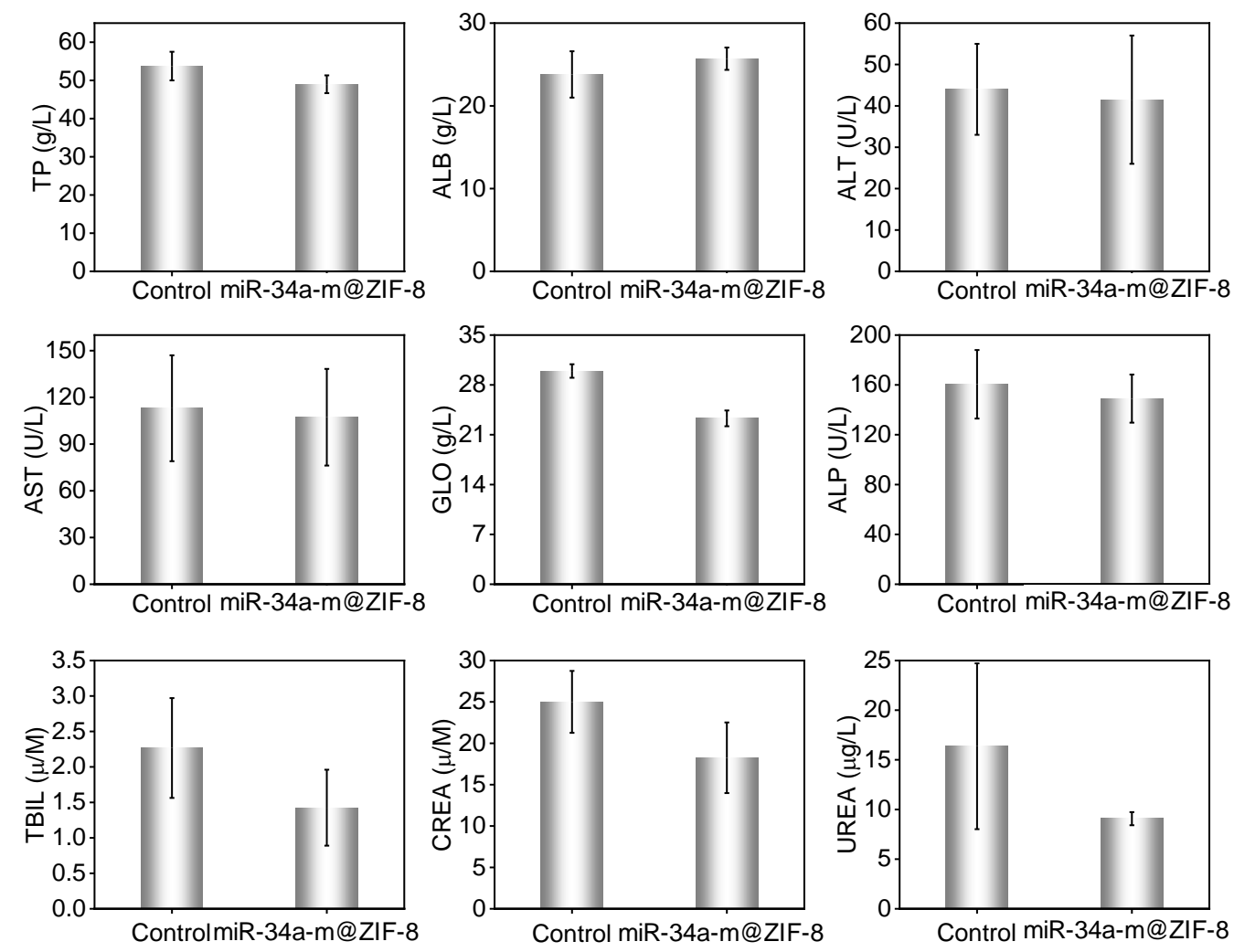

Figure S27. Blood biochemistry analysis of miR-34a-m@ZIF-8 treated mice with PBS treated as control.

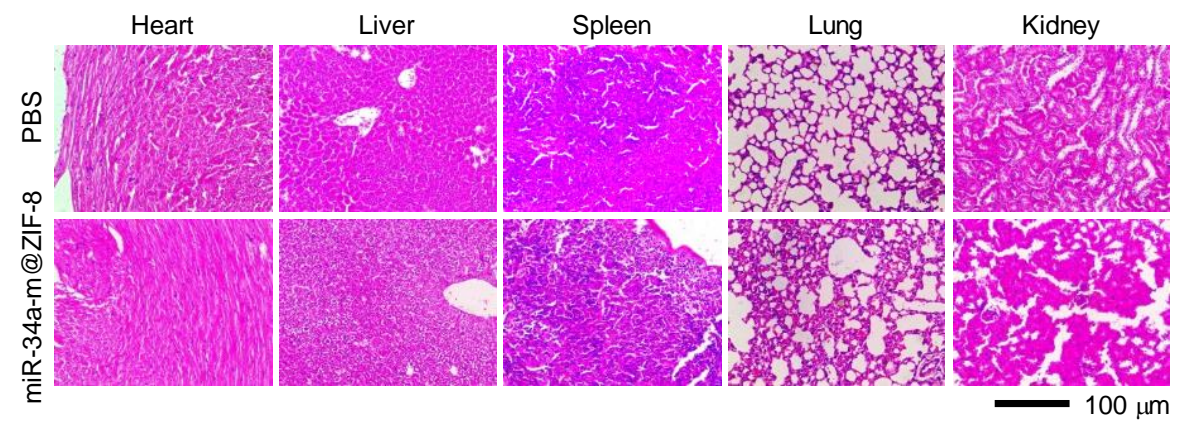

Figure S28. Representative H\&E staining of main organs of differently treated mice, including heart, liver, spleen, lung and kidney, which were collected from mice. 


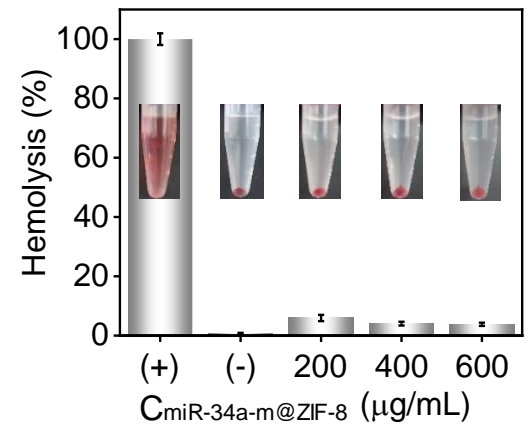

Figure S29. Hemolysis rates of miR-34a-m@ZIF-8 with water as positive control (+) and normal saline as negative control (-).
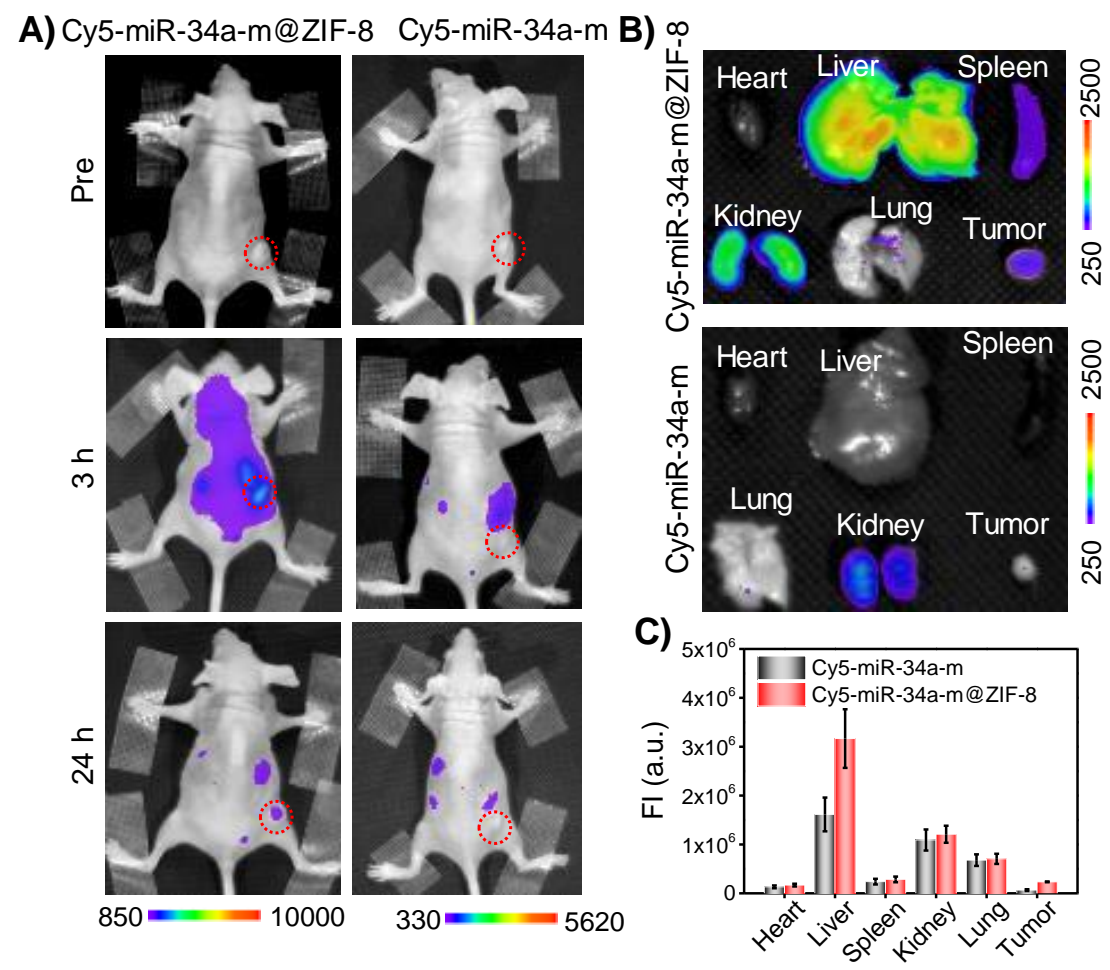

Figure S30. A) In vivo fluorescence images of MDA-MB-231 tumor-bearing nude mice after the injection of Cy5-miR-34a-m@ZIF-8. B) Ex vivo fluorescence images of major organs and tumors excised from the mice after injection for 24 hours. C) The corresponding fluorescence intensity of organs in B). 

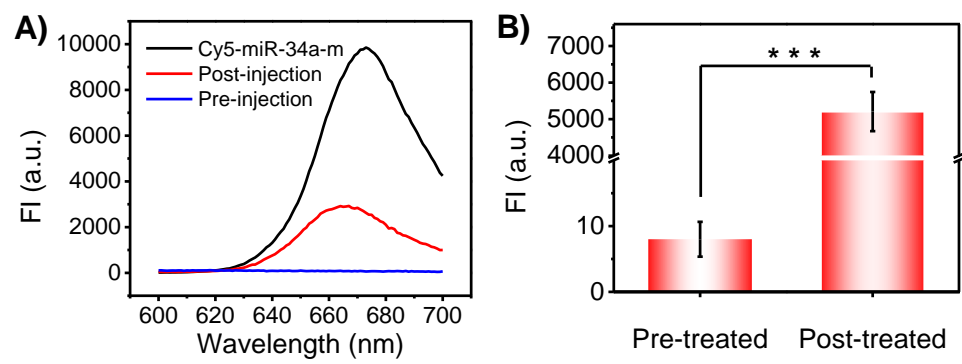

Figure S31. Cy5-miR-34a-m contents in the urine of Cy5-miR-34a-m@ZIF-8 treated mice in 12 h. (A) Fluorescence spectra of urea sample from mice post- and pre- injected with Cy5miR-34a-m@ZIF-8, and Cy5-miR-34a-m. (B) Corresponding fluorescence intensity.

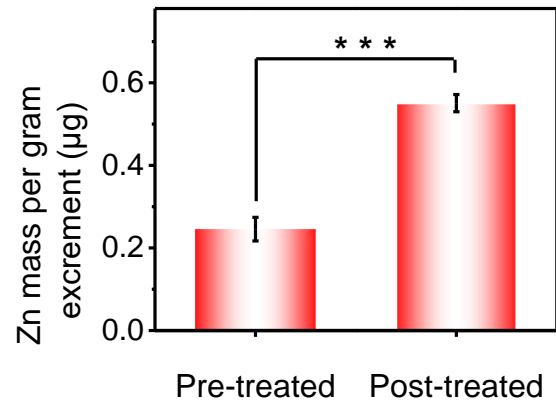

Figure S32. Zn contents per gram excrement of miR-34a-m@ZIF-8 treated mice in 12 h determined by ICP.

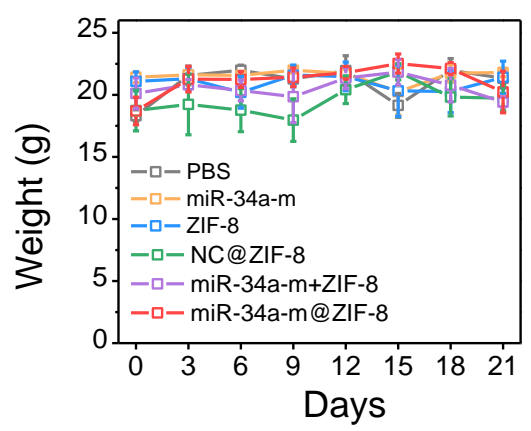

Figure S33. Body weight changes of treated mice. 


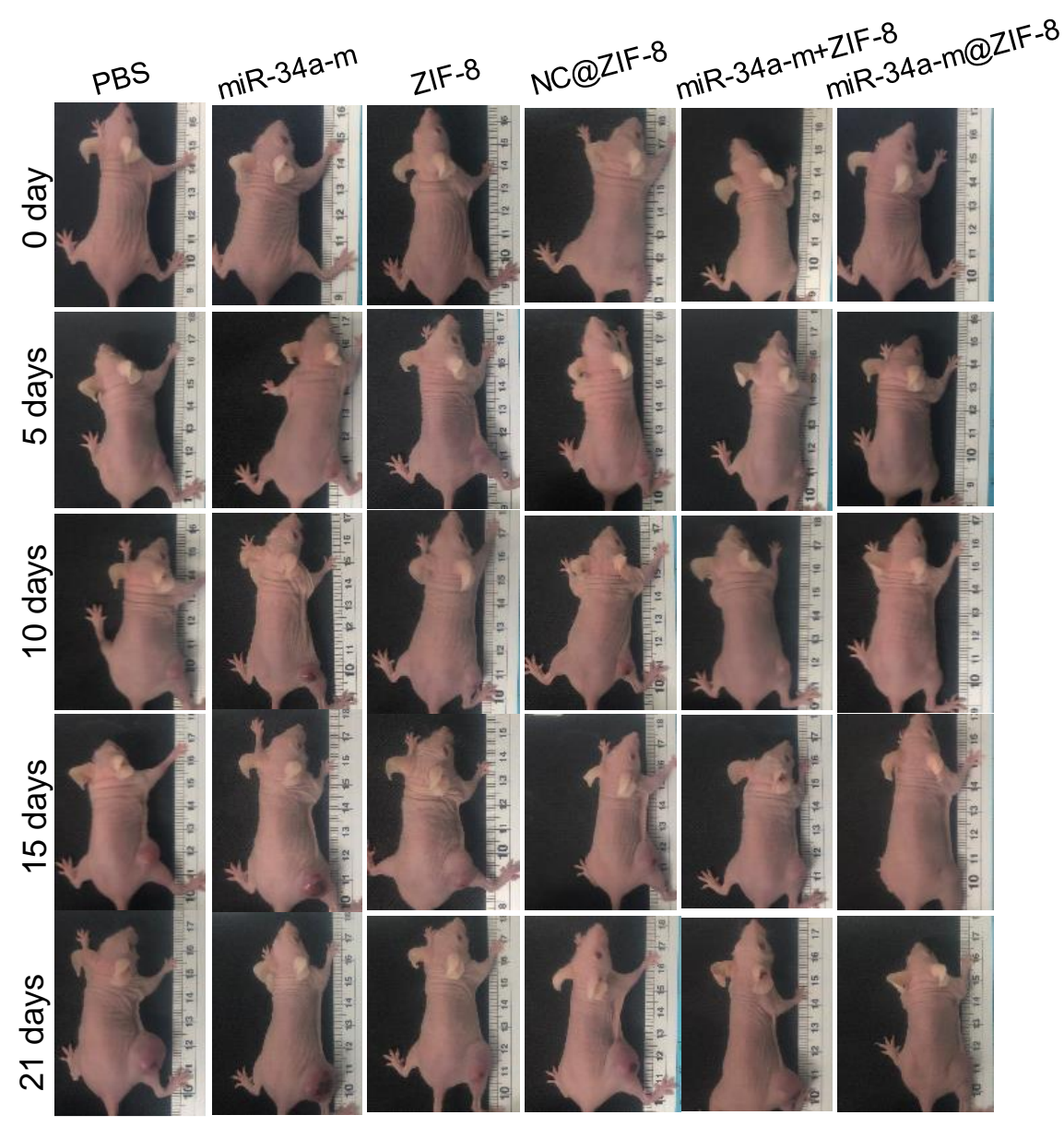

Figure S34. Photos of tumor-bearing mice intravenously injected with PBS, miR-34a-m, ZIF8, NC@ZIF-8, miR-34a-m + ZIF-8 (successive injection) and miR-34a-m@ZIF-8. 